\title{
Clinical governance in practice
}

\section{Femi Oyebode, Nick Brown \& Liz Parry}

Clinical governance is defined by the government as:

"a framework through which [National Health Service (NHS)] organisations are accountable for continuously improving the quality of their services and safeguarding high standards of care by creating an environment in which excellence in clinical care will flourish" (emphasis not in original) (Department of Health (DOH), 1998).

The intention is to re-assert the importance of quality of care within the management culture of the NHS. The Government's vision is of a structured and coherent approach to quality of care, even though the component parts of clinical governance, as described by the DOH (1998), are disparate and multifold. According to guidance, clinical governance will embrace a comprehensive programme of quality improvement activity such as: evidence-based practice and clinical audit; risk management programmes; professional performance procedures including continuing professional development (CPD); and other programmes including workforce planning. Clearly, much is expected of clinical governance.

Additional guidance on the implementation of clinical governance was published in March 1999 (DOH, 1999). In this document, the Government indicated that clinical governance is a developing concept which is very much in gradual evolution. It also set out a vision for the next five years and described the minimum requirements which have to be met in the year 1999/2000 (at the latest by April 2000). These are: to establish leadership; to carry out baseline assessments; to formulate and agree a development plan; and to clarify reporting arrangements for clinical governance within board and annual reports. With respect to establishing leadership, trusts are required to identify lead clinicians and to set up clinical governance committees as sub-committees of the board (see Box 1).

There was a requirement that a baseline assessment should be carried out at the latest by September 1999. The process and timescale for this had to be agreed with NHS regional offices. There are already a number of tools which allow trusts to evaluate their capacity to set up clinical governance. The

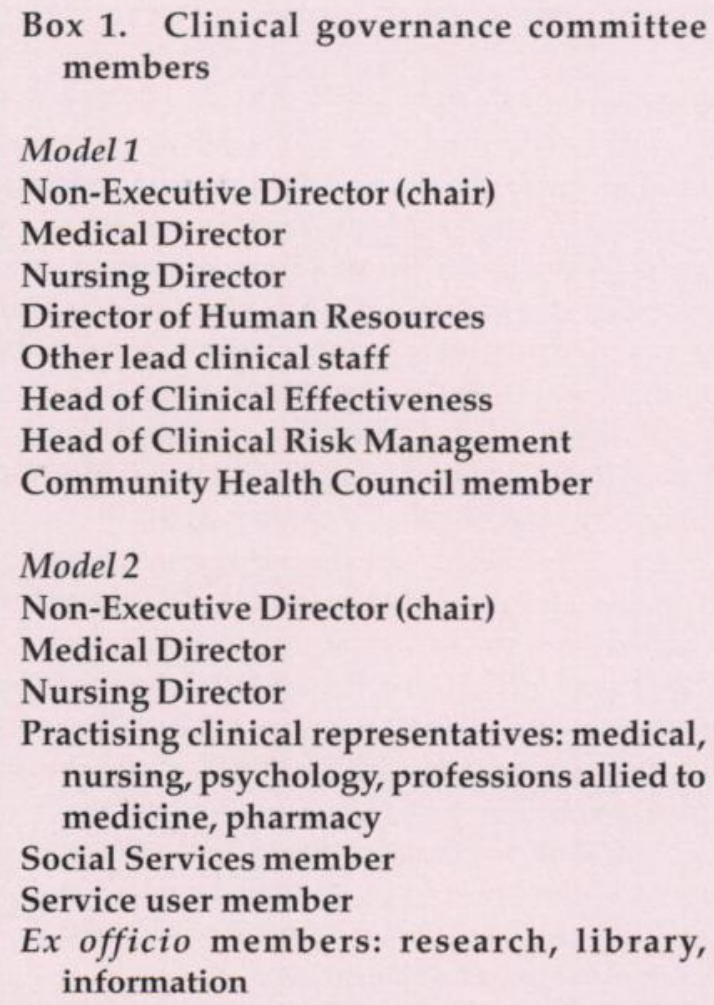

Box 1. Clinical governance committee members

Model 1

Non-Executive Director (chair)

Medical Director

Nursing Director

Director of Human Resources

Other lead clinical staff

Head of Clinical Effectiveness

Head of Clinical Risk Management

Community Health Council member

Model 2

Non-Executive Director (chair)

Medical Director

Nursing Director

Practising clinical representatives: medical, nursing, psychology, professions allied to medicine, pharmacy

Social Services member

Service user member

Ex officio members: research, library, information

Femi Oyebode is Professor of Psychiatry and Medical Director at South Birmingham Mental Health Trust, Vincent Drive, Edgbaston, Birmingham B15 2TZ. He has an interest in the development of measurement tools for evaluating clinical knowledge and skills in undergraduate, postgraduate and continuing medical education contexts. Nick Brown is Medical Director at Solihull Healthcare Trust. He is interested in postgraduate medical education and the development of methods of appraising the work of consultants with a view to developing personal development plans. Elizabeth Parry is the Management Assistant to Femi Oyebode, with responsibility for coordinating the implementation of a number of the component parts of clinical governance. She has an interest in the practical application of the concept of clinical governance. 
British Association of Medical Managers, for example, has developed a check-list for clinical governance which focuses on whether or not robust processes exist to support the systems described in the DOH's (1998) guidance (British Association of Medical Managers, 1998). In the West Midlands, the regional office has devised a structured system for evaluating trusts' capacities. This system assesses the overall approach to clinical governance. The framework for the evaluation is on five dimensions termed structure, process, outcome, culture and knowledge management (SPOCK).

The expectation is that once baseline assessments are completed, a development plan will be formulated and agreed with the regional offices. The plan may include developing infrastructure, identifying and responding to staff and board development needs, and agreeing the timescale for these activities. Finally, the reporting arrangements will need to be clarified.

\section{Quality assurance}

The guidance already published by the Government avoids discussion about the methodology of quality assurance programmes and does not concern itself with the specific agenda items that committees may wish to discuss or reports that committees may wish to receive. The authors' thinking on the following has been influenced by the work of the Joint Commission on the Accreditation of Health Care Organisations (JCAHO), an independent organisation in the USA that is involved in developing and monitoring standards of clinical care.

There are several component parts of quality within health care institutions. The quality of care provided by a doctor is central to any discussion about quality. There are at least two aspects to the performance of a doctor: the quality of technical performance and the quality of interpersonal performance.

Technical performance is dependent on the knowledge, judgement and skills necessary for recognising clinical conditions and developing treatment strategies. It is judged against a standard of best practice. It is recognised that even if the outcome for a given patient is poor, practice will be judged as good if it conforms to the standards believed to be the most effective for similar cases. The development of criteria for evaluating standards of practice must, therefore, be based on generally accepted practice standards. We will return to this issue below.

The interpersonal performance of clinicians is particularly important to patients. It is thought that patients tend to judge the quality of care by the interpersonal aspects of the process of care. There is little doubt that there is great difficulty in evaluating the interpersonal aspects of care, especially in psychiatry where the patient's mental state may influence his or her perception of events. None the less, patient preferences and opinions are important aspects of the overall quality of care and will have to be taken into account in some way.

The structure of the environment in which care is given is also an important aspect of quality. The physical environment, the number of staff and their qualifications, and the overall milieu of the institution are all important aspects of the care provided. Data summarising clinician technical and interpersonal performance and the quality of the structures should be received by the committee regularly. The question is, which criteria should be used to evaluate standards of care in a way that is acceptable to clinicians and understandable and meaningful for the purposes of the clinical governance committee?

There are a number of terms used in quality assurance which need to be defined, including norms, criteria, standards, indicators and sentinel events. 'Norms', for these purposes, are:

\footnotetext{
"measures of the actual clinical practice of health care professionals - statistical summaries of large numbers of patients without any implied judgement about the appropriateness of care. The average amount of a tricyclic antidepressant medication prescribed to patients with a diagnosis of major depressive disorder would be an example of a clinical norm in psychiatry" (Fauman, 1989).
}

'Criteria' are statements that define appropriate clinical care. An example of a quantitative criterion is defining the therapeutic dose of an antidepressant such as fluoxetine as $\mathbf{2 0 ~ m g}$ daily in an individual with a diagnosis of major depressive disorder. Criteria can be classified in other ways. For example, in relation to electroconvulsive therapy (ECT) there may be criteria relating to: (a) the structure and technical resources available to deliver ECT safely; (b) the actual process of administering ECT; and (c) the outcome of the care given. 'Standards' is a term that is used interchangeably with criteria. However, it can have the additional, perhaps confusing, connotation of being a quantitative specification of a criterion. For example, standards for ECT could be defined as the fulfilment of set criteria $80 \%$ or more of the time.

The term 'indicator' is another term in common parlance. It is described as a well-defined, measurable variable related to the provision or outcome of medical care. Unlike criteria, indicators are valuefree dimensional variables, in the sense that no judgement of good or ill attaches to them, although frequently they measure complications of care. In 
psychiatry, indicators could be the number of cases of tardive dyskinesia following the use of neuroleptics, or the number of violent incidents on an inpatient unit. Indicators as defined by the JCAHO relate to important dimensions of care that are highvolume, high-risk or problem-prone (JCAHO, 1986). An example might be the use of restraint. The valuefree nature of indicators means that a decision needs to be made about how to interpret any data about them. The idea is that a threshold should be agreed above which an evaluation must be triggered to determine whether or not a problem or opportunity to improve services exists. Thus, if the indicator rate rises above a certain level, an investigation would automatically be mounted to examine the clinical practice to which the indicator relates. For example, if discharges against medical advice were to be regarded as indicators, percentages of such discharges above a set level, say $25 \%$, would trigger intensive study.

'Sentinel events' are single-event indicators which by themselves trigger investigations. In psychiatry, sentinel events could include completed suicide, attempted suicide, self-mutilation, unauthorised absence, sexual abuse, adverse drug reaction, serious medication error, serum drug level above the therapeutic range, assault with injury to another, and transfer to an acute medical service. Initiation of medical negligence litigation could also act as a sentinel event. Peer review of the management of the case involved in a sentinel event could then take place. See Box 2 for the JCAHO's 10-step monitoring and evaluation model.

\section{Clinical audit}

Clinical audit is regarded as one of the cornerstones of clinical governance. There is a requirement that all hospital doctors will fully participate in audit programmes including speciality and sub-speciality national external audit programmes. The subject of the national confidential inquiries is already determined, but there is little guidance on what principles should underpin local audits, except that they should be aimed at improving clinical care. There is, of course, an implicit desire for clinical audit to identify poorly performing doctors. The public's understanding of the purpose of clinical audit has been influenced by the Bristol case where it has been widely claimed that if only the results of clinical audit had been attended to, the tragic situation would at least have been controlled earlier than was the case (Keogh et al, 1998). The truth about clinical audit, in contemporary practice, is that it is hardly a coherent venture with clearly expressed aims,
Box 2. Monitoring and evaluation model (see Taylor, 1992)

1 Assign responsibility for monitoring and evaluation activities

2 Delineate scope of care

3 Identify important aspects of care

4 Identify indicators related to those aspects of care (pre-determined levels and/or patterns of care or outcomes based on hospital performance over time or comparison to external standards or norms)

5 Establish thresholds for evaluation related to the indicators

6 Collect and organise data

7 Evaluate care when thresholds are reached

8 Take actions to improve care

9 Assess the effectiveness of the actions and document improvement

10 Communicate relevant information to the organisation-wide quality assurance programme

methods and valid findings. The topics chosen for audits are quite often fortuitous rather than planned and rationally justifiable.

The JCAHO has set out explicit audit programmes which focus on those functions and aspects of patient care that are essential to quality patient care and the environment. The standards that are audited are deliberately designed to be applicable to all organisations and across all services and derive from consensus among practitioners about what can be expected. These standards state their objectives and are easy to survey (see: www.jcaho.org/Perfmeas/ Std.htm). For example, there are a number of standards that are designed to measure the care that patients with a diagnosis of major depressive disorder receive. These include the following measures (see Box 3 for an example):

(a) number of adequate antidepressant medication trials for patients with major depression;

(b) assessment of a patient's potential for harm to self or others;

(c) documentation of risks and benefits of new treatment options discussed with patient;

(d) identification and supportive documentation of symptoms of major depression;

(e) number of depressed patients with no change in target symptoms after receiving treatment;

(f) changes in treatment plan when there is no change in target symptoms after receiving treatment;

(g) selection and documentation of ECT; 
Box 3. Example of a performance measure

\section{Clinical performance \\ Depression}

Performance measure

Number of adequate antidepressant medication trials of patients with major depression

Type of measure

Process

Focus of measure

Monitoring adequate trial(s) of antidepressant medications

Rationale

Psychotropic medications comprise one of the largest classes of prescription drugs. Since this class of medications is prescribed extensively by both psychiatrists and family physicians, assurance of proper prescribing practices has major health implications

Authorities note that more than $10 \%$ of patients needing antidepressants have received inadequate dosage and duration of pharmacotherapy. Authorities specifically note that a substantial percentage of patients with major depression, treated by psychiatrists, receive less than optimal antidepressant treatment

(h) number of in-patients with a depressive disorder who have terminated treatment against medical advice; and

(i) number of depressed patients with a suicide attempt or completed suicide (see: $w w w$.jcaho. org/Perfmeas/Nlhi/Profiles/).

The aim would be for these standards to be audited regularly, perhaps quarterly. Results of such audits could form the basis of clinical audit reports to the clinical governance committee. Differences between consultant teams and deviations from thresholds set by the committee would then trigger further analysis of performance. A rolling programme of audit such as this requires resources, as well as cooperation from clinicians, to succeed.

\section{Risk management}

Risk management is concerned with identification of the risks that exist, assessment of those risks for potential frequency and severity, elimination of some risks, and reduction of the effect of those that cannot be eliminated (NHS Executive, 1994). Risk management thus aims to reduce risk and this can effectively be achieved through the delivery of highquality care. Ensuring that clinical staff are competent to practise is a self-evident method of assuring the quality of clinical care. Robust selection arrangements assist in the appointment of appropriately qualified and skilled professionals. It is then essential that clinical staff have a proper understanding of their role in relation to the role of the other members of the multi-disciplinary team. The American Psychiatric Association has provided guidelines for psychiatric practice in public sector psychiatric facilities (www.psych.org/pract_of_psych/public_pract. $\mathrm{html})$. These guidelines emphasise that the care of acutely and persistently mentally ill patients is a specialised area that requires a high level of expertise. They provide model job descriptions for psychiatrists that list both the clinical and managerial responsibilities of psychiatrists. The psychiatrist is reminded that he or she has responsibility for providing direct psychiatric services through comprehensive evaluation, diagnosis, treatment planning, and treatment of patients. The guidelines continue with a clear statement of proper psychiatric and other medical evaluation and treatment of patients. This acts as a reminder of the need for, among other things: medical screening and review of histories to ensure the full range of medical/surgical factors is taken into account in determining diagnosis and treatment; direct evaluation of the patient before the prescription of psychotropic medication; and frequent review of patients. These guidelines are in marked contrast to the job descriptions currently issued to prospective consultants in the UK, which tend to describe the nature of the trust, the catchment area and the resources available, without specifying the actual role and responsibility of the consultant. The issue at stake here is the need for clearly understood roles in order to reduce the likelihood of errors or omissions in clinical practice. Continuing education and training also has a critical part to play in ensuring that clinical staff are competent to practise, and this is discussed in more detail below. Other aspects of risk management include clinical audit as described above and processes for evaluating untoward incidents, complaints, clinical negligence and litigation.

\section{Lifelong learning}

Clinical governance brings into sharp relief the responsibilities not only of organisations but also 
of individual practitioners. Although it is clear that individual doctors have always striven to maintain and, indeed, to improve the quality of clinical care, it is now well-understood that there is a need for organisations such as trusts to demonstrate explicitly that an individual practitioner is capable of continuing competent practice. In other words, the simple facts of having fulfilled the requirements for full registration with the General Medical Council and appointment as a consultant will no longer, on their own, vouchsafe continuing clinical competence. The Royal College of Physicians (1999) has highlighted the key components of its strategy, and these include: an annual two-way job plan review; the introduction of continuing medical education based on a personal development plan; involvement in local or national audit of clinical outcome and process; the introduction of a Royal College of Physicians standards adviser for each trust; and the piloting of multi-disciplinary peer assessment service reviews of clinical teams. The American Medical Association has an accreditation programme that is intended to deliver benefits to physicians, hospitals, employers and consumers alike but that is not simply a statement of minimum standards or verification of credentials. The system is comprehensive, encompassing ethics, peer review, technical clinical performance and patient satisfaction. In essence, the Royal College of Physicians and American Medical Association both describe a system which documents how a doctor keeps up to date throughout a working life, and which measures performance against practice standards and peer review. Various terms are used for the outcome of this process, including re-certification and revalidation.

Here, we will concentrate on the use of appraisal mechanisms and performance review as a prelude to formulating personal development plans. The Standing Committee on Postgraduate Medical and Dental Education (SCOPME) describes appraisal as a confidential, educational review process which assists reflection on personal, educational jobrelated achievements (SCOPME, 1998). What is important here is that the emphasis of appraisal is on education. Yet, there is a need for performance review during which doctors are encouraged and assisted to reflect on and analyse their own performance. This would be informed by views of peers and also by nationally published standards of practice. The aim would be for these reviews to prompt doctors to take action to capitalise on their strengths and remedy any weaknesses through appropriate educational or learning opportunities (SCOPME, 1999).

In practical terms, each consultant will meet annually with his or her medical or clinical director.
The aim of the meeting would be to discuss:

(a) the consultant's job plan, including other professional commitments such as work on College committees;

(b) the doctor's CPD activities, including meetings attended and credits attained;

(c) evidence of participation in audit; and

(d) review of performance in comparison to peers and nationally published standards.

At the end of the meeting there should be an agreed personal development plan that is individualised and responsive to the needs of a particular doctor. It is likely that the evidence which forms the basis of the discussion between the consultant and the medical director will be part of the portfolio of evidence submitted to the General Medical Council for re-validation purposes.

What is missing at present is a nationally agreed valid instrument for collecting the evidence needed for appraisal and performance review. Obligatory CPD is not without its critics. It is acknowledged that the public has a right to be protected from incompetent practitioners - what is doubtful is whether mandatory educational programmes are necessary or sufficient to ensure that result. There is little doubt that the mere attendance of an approved meeting for a defined duration is no guarantee of learning, especially if the process at work is perceived as coercive. It is argued that there is no evidence that mandatory continuing education offers any guarantee that learning will take place, nor is there evidence that the acquisition of knowledge and skills will ensure that they will be applied to improve performance. The issue is not whether professional people know something, it is whether they will apply it. It is also important to emphasise that what may seem to be the result of deficiency of knowledge may be owing to inadequate equipment, lack of supervision, conflicting expectations and so on. There is a risk that doctors will end up being addressed as if they do not have a personal, vested interest in keeping up to date.

The mechanisms currently under discussion all seem to assume that it is the public that is demanding that doctors keep up to date. In fact, most doctors derive a sense of pride from being competent professional people working in particular services or organisations. In other words, identity and selfesteem are bound up with one's capacity to function competently at work. This must be taken into account whatever system of appraisal and review is developed. An over-emphasis on the measurement and evaluation of competence at the expense of selfreflection and self-review is not likely to be productive. Appraisal systems should occur in a context in which CPD is regarded as assisting in 
clarifying the profession's functions, mastering theoretical and technical knowledge and encouraging the use of best interventions and techniques, with the proviso that these will be modified or changed in due course. The primary responsibility for CPD should rest with the individual, and the methods of delivering continuing professional education should be cognisant of the needs of adult learners who are already proficient in their chosen area of practice.

\section{Conclusion}

Mechanisms for ensuring continuing improvements in the delivery of clinical care are a legitimate concern of the public. This is especially the case in the UK where the service is publicly funded. Clinical governance is a process whereby the structures and functioning of health care organisations are opened to scrutiny as a means of reassuring the public that these institutions are fit for the purpose of caring for people. There is a genuine opportunity to improve the overall quality of care of patients. The changes required to implement clinical governance need resources, both human and financial, to succeed. For the present, the emphasis has remained on the structural changes without much discussion about the financial implications of the process of clinical governance. None the less, clinicians now have the opportunity to use clinical governance as a ready tool to bring clinical concerns and quality issues centre stage.

\section{References}

British Association of Medical Managers (1998) Clinical Governance in the New NHS. Cheadle: BAMM.

Department of Health (1998) A First Class Service: Quality In The New NHS. London: Department of Health

- (1999) Clinical Governance: Quality in the New NHS. London: Department of Health.

Fauman, M. A. (1989) Quality assurance monitoring in psychiatry. American Journal of Psychiatry, 146, 1121-1130.

Joint Commission for the Accreditation of Health Care Organisations (1986) Monitoring and evaluating the appropriateness of care: a hospital example. Quality Review Bulletin, 12, 326-330.

Keogh, B. E., Dussek, J., Watson, D., et al (1998) Public confidence and cardiac surgical outcome. British Medical Journal, 316, 1759-1760.

NHS Executive (1994) Risk Management in the NHS. London: Department of Health.

Royal College of Physicians (1999) Physicians Maintaining Good Medical Practice: Clinical Governance and Self-Regulation. London: Royal College of Physicians.

Standing Committee on Postgraduate Medical and Dental Education (1998) Continuing Professional Development for Doctors and Developments: Recommendations for Hospital Consultant CPD and Draft Principles for all Doctors and Dentists. London: SCOPME.
- (1999) Doctors And Dentists: The Need for a Process of Review. London: SCOPME.

Taylor, D. (1992) The Joint Commission Quality Assessment and Improvement Model. In Manual of Psychiatric Quality Assurance (ed. M. R. Mattson), pp. 69-77. Washington, DC: American Psychiatric Association.

\section{Multiple choice questions}

1. Clinical governance:

a is a framework for continuously improving the quality of clinical services

b includes as a component part risk management programmes

c is aimed solely at doctors

$\mathrm{d}$ is a method of assessing the effectiveness of clinical practice

e will allow trusts to decide which medical conditions to treat.

2. An 'indicator' in quality assurance:

a measures of the day-to-day practice of clinicians

$b$ is the measure of poor outcome in clinical practice

c is a value-free dimensional variable relating to clinical practice

$\mathrm{d}$ is the threshold for determining whether to investigate an event fully

e is a pre-set criterion of good clinical practice.

3. Sentinel events:
a are single event indicators
b can trigger investigations on their own
c could include completed suicide
d are synonymous with clinical negligence
e are an indication of poor practice.

4. Appraisal of consultants will involve:
a annual review of job plans
b successful completion of examinations of competence
c producing evidence of continuing profes- sional development
d presentation at trust board meetings
e review of performance in comparison to peers.

MCQ answers

$\begin{array}{lllllllll}\text { 1 } & & \text { 2 } & & \text { 3 } & & 4 & \\ \text { a } & \text { T } & \text { a } & \text { F } & & \text { a } & \text { T } & \text { a } & \text { T } \\ \text { b } & \text { T } & \text { b } & \text { F } & \text { b } & \text { T } & \text { b } & \text { F } \\ \text { c } & \text { F } & \text { c } & \text { T } & \text { c } & \text { T } & \text { c } & \text { T } \\ \text { d } & \text { F } & \text { d } & \text { F } & \text { d } & \text { F } & \text { d } & \text { F } \\ \text { e } & \text { F } & \text { e } & \text { F } & \text { e } & \text { F } & \text { e } & \text { T }\end{array}$

